\title{
Resting Glutamate Levels and Rapid Glutamate Transients in the Prefrontal Cortex of the Flinders Sensitive Line Rat: A Genetic Rodent Model of Depression
}

\author{
Kevin N Hascup 1,4, Erin R Hascup ${ }^{1,4}$, Michelle L Stephens², Paul EA Glaser², Takashi Yoshitake', \\ Aleksander A Mathé ${ }^{3}$, Greg A Gerhardt ${ }^{2}$ and Jan Kehr*,I \\ 'Department of Physiology and Pharmacology, Karolinska Institutet, Stockholm, Sweden; '2Department of Anatomy \& Neurobiology, Center for \\ Microelectrode Technology, Morris K Udall Parkinson's Disease Research Center of Excellence, University of Kentucky, Lexington, KY, USA; \\ ${ }^{3}$ Department of Clinical Neuroscience, Karolinska Institutet, Stockholm, Sweden
}

\begin{abstract}
Despite the numerous drugs targeting biogenic amines for major depressive disorder (depression), the search for novel therapeutics continues because of their poor response rates $(\sim 30 \%)$ and slow onset of action ( $2-4$ weeks). To better understand role of glutamate in depression, we used an enzyme-based microelectrode array (MEA) that was selective for glutamate measures with fast temporal $(2 \mathrm{~Hz})$ and high spatial $(15 \times 333 \mu \mathrm{m})$ resolution. These MEAs were chronically implanted into the prefrontal cortex of 3-to 6-month-old and 12- to 15-month-old Flinders Sensitive Line (FSL) and control Flinders Resistant Line (FRL) rats, a validated genetic rodent model of depression. Although no changes in glutamate dynamics were observed between 3 and 6 months FRL and FSL rats, a significant increase in resting glutamate levels was observed in the 12- to 15-month-old FSL rats compared with the 3-to 6-month-old FSL and age-matched FRL rats on days 3-5 post-implantation. Our MEA also recorded, for the first time, a unique phenomenon in all the four rat groups of fluctuations in resting glutamate, which we have termed glutamate transients. Although these events lasted only for seconds, they did occur throughout the testing paradigm. The average concentration of these glutamate-burst events was significantly increased in the 12- to I5-month-old FSL rats compared with 3- to 6-month-old FSL and age-matched FRL rats. These studies lay the foundation for future studies of both tonic and phasic glutamate signaling in rat models of depression to better understand the potential role of glutamate signaling in depression.

Neuropsychopharmacology (20 II) 36, 1769-1777; doi:10.1038/npp.201 I.60; published online 27 April 20I I
\end{abstract}

Keywords: electrode; biosensor; freely moving; antidepressant; basal glutamate; major depressive disorder

\section{INTRODUCTION}

Major depressive disorder (depression) is a chronic, recurring mental illness affecting $\sim 340$ million people worldwide and is considered to be an underlying cause in $35-40 \%$ of suicides (Kulkarni and Dhir, 2009). Depression is characterized by lowered mood and loss of interest in daily activities leading to social, occupational, and educational impairments. These impairments cause decreased quality of life, decreased productivity, and increased health-care costs (Skolnick et al, 2009). Children, adolescents, and young adults, who have significant risk of recurrence,

*Correspondence: Dr J Kehr, Department of Physiology and Pharmacology, Nanna Svartz väg 2, Karolinska Institutet, SE-I7I 77 Stockholm, Sweden, Tel: +4685248 7084, Fax + 46852487234 , E-mail: Jan.Kehr@ki.se

${ }^{4}$ These authors contributed equally to this work.

Received 28 October 2010; revised I4 March 2011; accepted I5 March 201। are increasingly diagnosed with the disorder (McCauley et al, 1993).

Depression treatments include pharmacotherapy, psychotherapy, magnetic therapy, and electroconvulsive methods. Pharmacological treatments often target biogenic amines, but despite the plethora of commercially available antidepressants, the search for novel therapeutic targets and specific drugs is an ongoing endeavor for several reasons: (1) Most depressed patients are not adequately treated with monotherapy. Placebo-controlled trials show efficacy rates of $\sim 30 \%$, and few people achieve total remission of depressive symptoms despite using multiple therapies (Rakofsky et al, 2009). Recent data from the STAR ${ }^{\star} \mathrm{D}$ trial revealed remission rates of $\sim 30 \%$ for citalopram monotherapy, and this only increased to $\sim 50-70 \%$ with the most intensive combination of therapies (Insel and Wang, 2009). (2) Current antidepressants, even when effective, often are discontinued because of side effects, including sexual dysfunction, nausea, weight gain, and precipitation of a 
manic phase. (3) Current antidepressants have a slow onset of clinical response (2-4 weeks) that prolongs the suffering and can result in dire consequences for patients with suicidal ideation (Skolnick et al, 2009; Nierenberg, 2001). (4) All antidepressants currently carry a warning for their ability to increase suicidal thoughts in a small percentage of adolescents and young adults. Although this effect appears to lessen after the age of 25 years, it discourages some physicians from using antidepressants in adolescents (Stone et al, 2009).

Glutamate, the major excitatory neurotransmitter in the mammalian central nervous system, is implicated in several neurodegenerative and neuropsychiatric disorders (Danbolt, 2001). Recent clinical and post-mortem studies of depressed patients have found pathophysiological evidence of altered glutamatergic neurotransmission (Witkin et al, 2007). Depressed patients have increased serum and plasma levels of glutamate compared with controls (Mauri et al, 1998; Altamura et al, 1993; Kim et al, 1982), and the severity of the depression is correlated with increases in plasma glutamate levels (Mitani et al, 2006). Post-mortem brain tissue shows increased glutamate levels in depressed patients compared with controls (Hashimoto et al, 2007). This clinical data has lead to the development of several therapeutic strategies targeting glutamatergic receptors for the treatment of depression (Kugaya and Sanacora, 2005; Paul and Skolnick, 2003). Sub-anesthetic doses of ketamine, an $N$-methyl-D-aspartate receptor antagonist, have antidepressant effects with immediate onset of action (Maeng and Zarate, 2007; Berman et al, 2000) probably due to its ability to increase synaptogenesis ( $\mathrm{Li}$ et al, 2010). Riluzole inhibits presynaptic glutamate release and open label trials have shown clinical efficacy in the treatment of depression (Brennan et al, 2010). On the basis of this clinical evidence, a current hypothesis proposes that increased extracellular glutamate causes excessive glutamatergic neurotransmission that is deleterious to neuronal function and contributes to depression (McNally et al, 2008; Pittenger et al, 2007).

In preclinical settings, several animal models for depression have been developed (Pollak et al, 2010). Of those, the Flinders Sensitive Line (FSL) rat is one of several extensively validated genetic rodent models of depression with many traits similar to those observed in depressed individuals. Studies of mature (3-month-old) FSL rats support decreased appetite and weight when compared with control, Flinders Resistant Line (FRL) rats (Overstreet, 1993). FSL rats show high degrees of psychomotor retardation compared with FRL control littermates. They are less active in novel, openfield environments, and bar-presses at a lower rate for water or food reward (Overstreet and Russell, 1982). However, there was no evidence for anhedonia or cognitive disturbances (Overstreet et al, 2005) in FSL rats. Furthermore, FSL rats display severe immobility on the forced swim task (Kulkarni and Dhir, 2009; Overstreet et al, 1994) that is reversed with chronic treatment of antidepressants (Yadid et al, 2000). For a complete review of the studies conducted in this rat model, the reader is referred to Overstreet et al, 2005.

As alterations in glutamatergic neurotransmission may be an underlying cause in the pathology of depression, we decided to study resting glutamate levels in the FSL rats compared with the control FRL rats. We used an enzyme-based microelectrode array (MEA), selective for measuring glutamate with low limits of detection $(<0.2 \mu \mathrm{M})$, fast temporal resolution $(500 \mathrm{~ms})$ (Burmeister et al, 2000, 2002, 2004; Burmeister and Gerhardt, 2001) and minimal damage to surrounding brain tissue $(50-100 \mu \mathrm{m})$ (Hascup et al, 2009). Using these MEAs in combination with pharmacological treatments, we have shown that resting glutamate levels in the medial prefrontal cortex (PFC) are at least $50 \%$ neuronally derived. Furthermore, preapplication of tetrodotoxin (TTX) completely abolishes the physiological glutamate response to a tail-pinch stressor (Hascup et al, 2010). These MEAs were chronically implanted into the PFC of young (3- to 6-month) and old (12- to 15-month) FSL and FRL rats allowing us to measure extracellular levels of resting glutamate over several days (Hascup et al, 2008; Rutherford et al, 2007) in a clinically relevant brain structure implicated in depression (Canbeyli, 2010).

\section{MATERIALS AND METHODS}

\section{Animals}

A colony of FSL and FRL rats were maintained at the Department of Physiology and Pharmacology, Karolinska Institutet, Stockholm, Sweden. Male rats were separated into 3- to 6-month (young, FRL $n=7$; FSL $n=6$ ) and 12- to 15-month (old, FRL $n=4$; FSL $n=4$ ) age groups for all experiments. Male Fischer-344 rats were separated into 3- to 6 -month-old $(n=7)$ and 18-month-old $(n=6)$ age groups. All animals were group-housed in a $12 \mathrm{~h}$ light/dark cycle at a constant room temperature of $23^{\circ} \mathrm{C}$ with ad libitum access to food and water. Animals were treated in accordance with protocols approved by the Animal Ethics Committee of Stockholm, the Federation of European Laboratory Animal Science Associations, and all procedures were conducted in conformity with the Karolinska Institutet's Guidelines for animal welfare. Efforts were made to minimize the number of animals used and reduce their sufferings. Animals were allowed at least 1 week to acclimate to the testing environment and to the researchers before any experiments. All appropriate animal care (food, water, bedding, cage cleaning, and so on) was performed by the animal resource center staff. Following surgery, rats were individually housed under the same conditions. Upon completion of the studies, rats were killed with an overdose of anesthetic. The brains were removed and sliced for visual inspection of MEA placement.

\section{Microelectrode Array Design}

MEAs were assembled and selected for in vivo recordings as previously described (Burmeister et al, 2002, 2000). Preparation of the MEA for recording has been extensively described in Hascup et al, 2006 and Burmeister et al, 2002. Briefly, the platinum recording sites were dip coated with Nafion (Sigma-Aldrich, St Louis, MO) to repel anions (Burmeister and Gerhardt, 2001). Two of the MEA recording sites were coated with an L-glutamate oxidase (EC 1.4.3.11) (Seikagaku America, East Falmouth, MA) coating solution (Nickell et al, 2005). To induce crosslinking of L-glutamate oxidase and to increase the adhesion to the microelectrode, glutaraldehyde, and BSA (Sigma-Aldrich) 
a

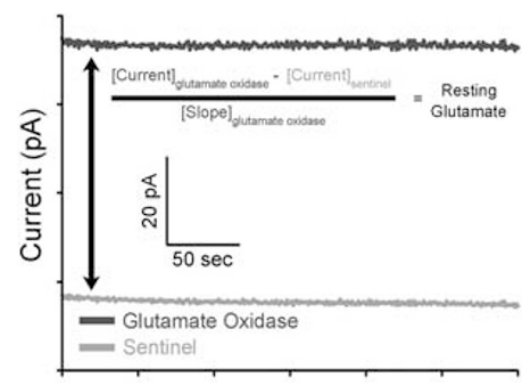

Time (seconds)

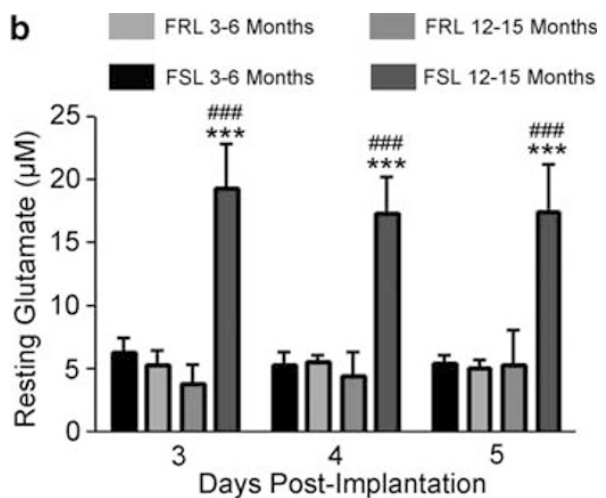

Figure I Resting glutamate Levels. (a) An amperometric trace of resting glutamate determination from a I2- to I5-month-old FSL rat. Resting glutamate levels were calculated from a 300 s baseline average, before burst analysis studies, using the equation shown in the figure. This baseline was calculated when little-to-no burst events were observed, thus, fluctuations from spontaneous increases in glutamate spillover did not arbitrarily inflate resting glutamate values. (b) Resting levels of glutamate in young and old FRL and FSL rats. A two-way ANOVA was used to compare resting glutamate levels in 3- to 6-month-old FRL $(n=7)$ and FSL $(n=6)$ rats, I2- to I5-month-old FRL $(n=4)$ and FSL $(n=4)$ rats and 3- to 6-month and I2- to I5-month-old FSL rats. A two-way ANOVA indicated no effect of recording day $(F=0.11$; $D F n=2$, DFd $=49 ; p=0.90)$, however, a significant effect of rat group was observed ( $F=42.92$; $\mathrm{DFn}=3, \mathrm{DFd}=49 ; p<0.000 \mathrm{I})$. A Bonferonni post-hoc revealed 12- to I5-month-old FSL rats had significantly elevated levels of resting glutamate compared with age-matched FRL rats (**** $p<0.001$ ) as well as 3- to 6-month-old FSL rats ( ${ }^{\# \#} p<0.001$ ).

were added to the L-glutamate oxidase solution. The L-glutamate oxidase layer was required by our technique to measure glutamate levels, as it causes the enzymatic breakdown of glutamate to $\alpha$-ketoglutarate and the electroactive reporter molecule, $\mathrm{H}_{2} \mathrm{O}_{2}$. When a potential of $+0.7 \mathrm{~V}$ vs a $\mathrm{Ag} / \mathrm{AgCl}$ reference electrode was applied, the reporter molecule, $\mathrm{H}_{2} \mathrm{O}_{2}$, was oxidized giving two electrons at the MEA recording surface. The resulting current was then amplified and recorded by a Mark II FAST16 recording system (Quanteon, LLC, Nicholasville, KY). The remaining two MEA recording sites (self-referencing or sentinel sites) were coated similar to the glutamate recording sites, with the exception that the coating solution did not contain Lglutamate oxidase. This meant the sentinel sites could not record any converted glutamate but could record any electroactive interferents (at $+0.7 \mathrm{~V} v s$ a $\mathrm{Ag} / \mathrm{AgCl}$ reference electrode) not repelled by Nafion. When the current recorded on the sentinel sites was subtracted from the current on the glutamate recording sites, the resulting signal represents the resting glutamate levels in brain tissue (Hascup et al, 2010; Burmeister et al, 2002; Burmeister and Gerhardt, 2001).

\section{Reference Electrodes}

A miniature $\mathrm{Ag} / \mathrm{AgCl}$ reference electrode was prepared by first stripping the Teflon off the silver wire $(200 \mu \mathrm{m}$ bare, $275 \mu \mathrm{m}$ coated; A-M Systems, Carlsberg, WA) $\frac{1}{4}$ inch on each end. One of the stripped ends was soldered to a gold-plated socket (Ginder Scientific, Ottawa, ON, Canada) and the other end was coated with $\mathrm{AgCl}$ by placing the tip of the stripped sliver wire (cathode) into a $1 \mathrm{M} \mathrm{HCl}$ plating bath saturated with $\mathrm{NaCl}$ containing a $\mathrm{Pt}$ wire (anode) and applying $+9 \mathrm{~V}$ DC using a power supply to the cathode $v s$ the anode for 5-10 min.

\section{Ceramic MEA Preparation}

The MEA paddle was modified for use in freely moving animals as extensively described in previous literature (Hascup et al, 2008; Rutherford et al, 2007, Hascup et al, 2006). Briefly, the pedestal was the portion of the system that was chronically implanted on the rat's head and contained the MEA. During recording sessions, the pedestal was connected to the miniaturized Rat Hat, containing the 4-channel amplifier that was in turn connected to a low torque commutator.

\section{MEA Calibration}

MEAs were calibrated to determine their sensitivity and selectivity against ascorbic acid as previously described (Hascup et al, 2010, 2009, 2008, 2006; Nickell et al, 2005). Selectivity ratios for glutamate over ascorbic acid were calculated in addition to the slope (sensitivity), limit of detection (LOD), and linearity $\left(R^{2}\right)$ for glutamate for all MEAs. For this study, we used 21 MEAs consisting of 37 active glutamate recording sites. The MEAs used in this study had an average glutamate sensitivity of $7.2 \mathrm{pA} / \mu \mathrm{M}$, selectivity ratio of $80: 1$, and signal-to-noise ratio of $0.2 \mu \mathrm{M}$.

\section{MEA Implantation}

MEA implantation has been extensively described in previous literature (Hascup et al, 2010, 2009; Rutherford et al, 2007). The MEA pedal assembly was implanted in the right PFC (MEA tip coordinates: AP: $+3.2 \mathrm{~mm}$; ML: $0.8 \mathrm{~mm}$, DV: $-5.0 \mathrm{~mm} v s$ bregma), with the incisor bar set so that the skull was level $(-2.3 \mathrm{~mm})$, which was based on the atlas of Paxinos and Watson (1998). Rats were allowed to recover for a minimum of 2 days before initial recordings.

\section{Recording Protocol}

Typical recording sessions involved allowing the rat to freely roam around the recording chamber for $30 \mathrm{~min}$ to acclimate to the surroundings before connecting the pedestal to the Rat Hat potentiostat and the start of data acquisition. Once the recordings were started, the rat underwent a minimum 90-min acclimation period, or until a stable baseline was established. Once a stable baseline was 
obtained, the recording continued for one additional hour that provided us with the necessary data for analysis of resting glutamate and fluctuations in glutamate spillover events or bursts.

\section{Data Analysis}

The Mark II FAST16 recording system saved amperometric data at $2 \mathrm{~Hz}$ for all four recording channels. Unless otherwise noted, calibration data, in conjunction with a MATLAB graphic user interface program developed by Jason Burmeister, LLC, was used to calculate resting glutamate levels and fluctuations in glutamate spillover. Resting glutamate was calculated by taking a $300 \mathrm{~s}$ baseline average, thereby, fluctuations from spontaneous increases in glutamate spillover did not arbitrarily increase resting glutamate levels (Figure 1a). To analyze rapid glutamate transients, the MATLAB graphic user interface was set to detect peaks above a signal to noise ratio of 2 with a moving baseline of 10 points (or $5 \mathrm{~s}$ ). A new baseline was measured before the start of each glutamate transient. Measures derived from the MATLAB graphic user interface file included: (1) resting glutamate $(\mu \mathrm{M}),(2)$ transient interval (seconds), (3) transient duration (seconds), and (4) transient maximum amplitude $(\mu \mathrm{M})$ from baseline. A two-way analysis of variance (ANOVA) followed by a Bonferonni posthoc analysis was used to analyze resting glutamate, transient interval, transient duration, and transient maximum amplitude over days. A one-way ANOVA followed by a Tukey's post-hoc was used to analyze the averages of transient interval, transient duration, and transient maximum amplitude on all days tested. A two-tailed $t$-test was used to compare the same parameters 3- to 6- and 18-month-old Fischer-344 rats and to age-matched FSL rats. Data are shown as mean \pm SEM and significance was defined as $p<0.05$.

\section{RESULTS}

\section{Resting Glutamate}

Resting glutamate levels were determined on days 3-5 postimplantation in the PFC of 3- to 6-month-old (young) and 12- to 15-month (old) FRL and FSL rats as shown in Figure $1 \mathrm{~b}$. As previously described, the self-referencing electrode current was subtracted from the glutamate recording electrode current (and divided by the glutamate recording site slope obtained from in vitro calibration). As shown in Figure $1 \mathrm{~b}$, consistent resting glutamate levels on days 3-5 post-implantation were seen for the 3- to 6-month-old FRL $(6.3 \pm 1.2 \mu \mathrm{M}, 5.3 \pm 1.0 \mu \mathrm{M}$, $5.4 \pm 0.6 \mu \mathrm{M} ; n=7)$ and FSL $(5.3 \pm 1.0 \mu \mathrm{M}, 5.5 \pm 0.5 \mu \mathrm{M}$, $5.0 \pm 0.6 \mu \mathrm{M} ; n=6)$ and the 12- to 15-month-old FRL $(3.8 \pm 1.4 \mu \mathrm{M}, \quad 4.4 \pm 1.8 \mu \mathrm{M}, \quad 5.2 \pm 2.7 \mu \mathrm{M} ; n=4)$ and FSL $(19.3 \pm 3.4 \mu \mathrm{M}, \quad 17.3 \pm 2.9 \mu \mathrm{M}, \quad 17.4 \pm 3.7 \mu \mathrm{M} ; n=4)$ rats. A two-way ANOVA revealed that the day post-implantation had no effect on resting glutamate levels $(\mathrm{F}=0.11 ; \mathrm{DFn}=2$, $\mathrm{DFd}=49 ; p=0.90)$, however, a significant effect of rat group was observed $(\mathrm{F}=42.92 ; \mathrm{DFn}=3$, $\mathrm{DFd}=49 ; p<0.0001)$, and no effect of interaction $(\mathrm{F}=0.2 ; \mathrm{DFn}=6, \mathrm{DFd}=49 ; p=0.98)$. Using a Bonferonni post-hoc, we compared resting glutamate levels between animal groups. There was no difference in resting glutamate levels between 3 and 6 months FRL and FSL rats as well as in the 3- to 6-month-old FRL and 12- to 15-month-old FRL rats. However, we did observe significantly
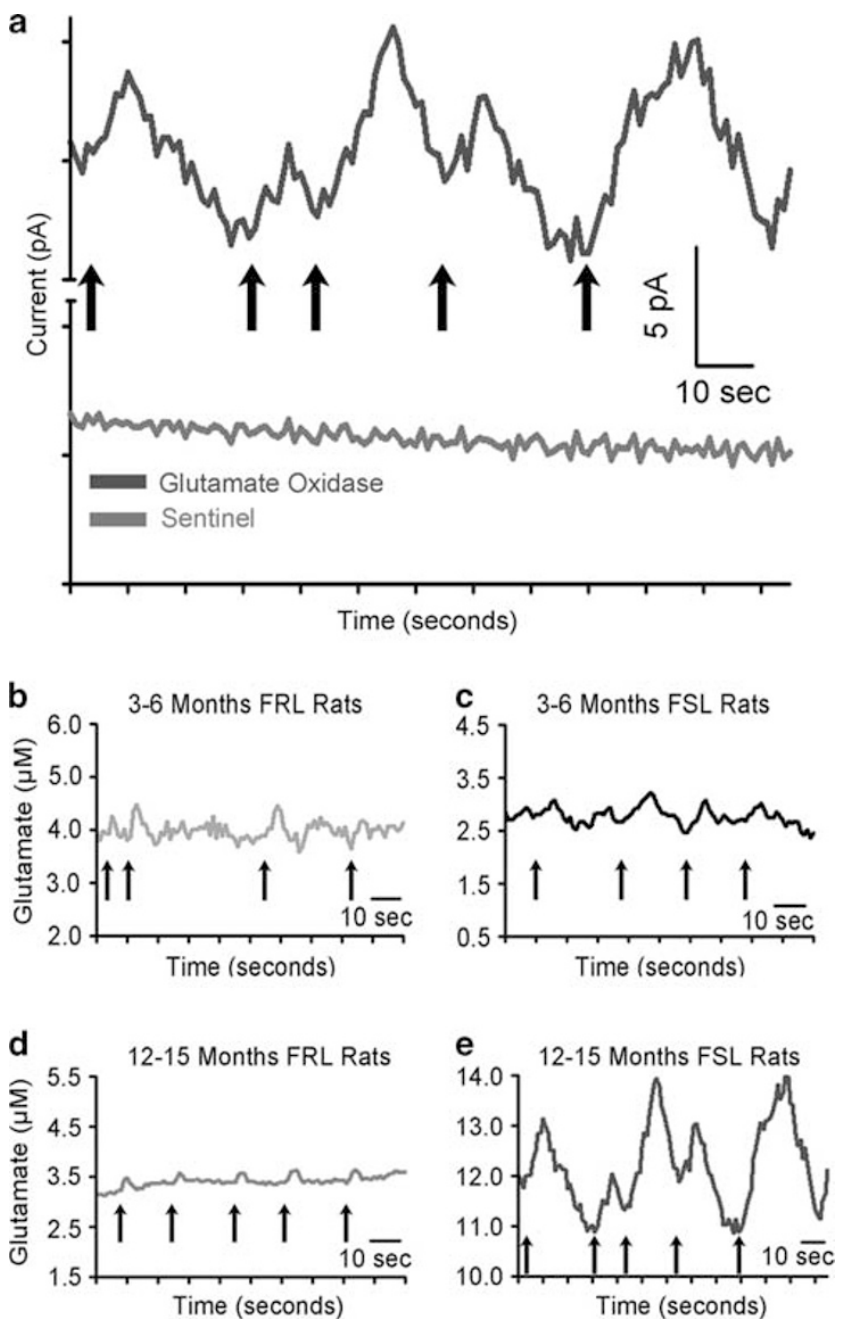

Figure 2 Rapid glutamate transients. (a) An amperometric trace of spontaneous glutamate bursts from the same 12- to 15-month-old FSL rat shown in Figure la. Rapid fluctuations in current were observed on the L-glutamate oxidase-coated site (blue) and not on the sentinel-recording site (green). Arrows indicate the start of a spontaneous burst spillover event, which was detected using the MATLAB graphic user interface. Note, the $y$ axis was segmented to emphasize these spontaneous release events only occurred on the glutamate oxidase-coated sites and not the selfreferencing sites. Self-referenced representative traces of increased glutamate bursts in 3- to 6-month-old FRL (b) and FSL (c), and the 12 to 15-month-old FRL (d) and FSL (e) rats. All rat groups displayed intermittent glutamate bursts with multiple events occurring in rapid succession. The increased glutamate spillover was similar in the 3- to 6-month-old FRL and FSL and the 12-to 15-month-old FRL rats, however, the 12-to 15-month-old FSL rats showed larger average concentrations of glutamate spillover. Arrows indicate the start of an increased glutamate spillover event.

increased $(p<0.001)$ resting glutamate levels in the 12 - to 15-month-old FSL rats compared with the 12- to 15-month-old FRL rats on all testing days. Also, 12- to 15-month-old FSL rats had significantly $(p<0.001)$ higher resting glutamate levels compared with 3- to 6-month-old FSL rats on all testing days.

\section{Rapid Glutamate Transients}

Rapid glutamate transients were observed throughout the duration of the tests in all rat groups, over all days tested. Figure 2a shows an amperometric trace highlighting these 
Table I Rapid Glutamate Transients in FRL and FSL rats

\begin{tabular}{cccccc} 
Age & \multicolumn{2}{c}{ 3- to 6-month-old } & & \multicolumn{2}{c}{ 12- to 15-month-old } \\
\cline { 2 - 3 } \cline { 5 - 6 } Rat & FRL & FSL & & FRL & FSL \\
$n$ & 7 & 6 & 4 & 4 \\
\hline
\end{tabular}

\begin{tabular}{|c|c|c|c|c|c|}
\hline \multirow{3}{*}{ Glutamate transients/min } & & & & & \\
\hline & \multicolumn{5}{|c|}{ Days post-implantation } \\
\hline & 3 & $1.8 \pm 0.3$ & $1.9 \pm 0.3$ & $2.1 \pm 0.5$ & $1.7 \pm 0.5$ \\
\hline & 4 & $1.8 \pm 0.2$ & $1.8 \pm 0.3$ & $1.8 \pm 0.7$ & $1.7 \pm 0.3$ \\
\hline \multirow[t]{2}{*}{ Glutamate transient interval ( $\mathrm{s} \pm$ SEM) } & 3 & $59.6 \pm 7.6$ & $56.6 \pm 3.1$ & $63.2 \pm 13.1$ & $94.8 \pm 23.5$ \\
\hline & 4 & $59.2 \pm 6.2$ & $52.0 \pm 1.1$ & $77.3 \pm 21.3$ & $88.2 \pm 17.0$ \\
\hline & 4 & $18.0 \pm 2.3$ & $13.2 \pm 2.9$ & $15.2 \pm 2.4$ & $33.6 \pm 7.8^{*}, \# \#$ \\
\hline & 5 & $18.2 \pm 2.4$ & $14.3 \pm 3.2$ & $15.4 \pm 2.9$ & $24.5 \pm 2.4$ \\
\hline \multirow[t]{3}{*}{ Glutamate transient amplitude $(\mu \mathrm{M} \pm \mathrm{SEM})$} & 3 & $0.3 \pm 0.1$ & $0.3 \pm 0.1$ & $0.5 \pm 0.1$ & $1.6 \pm 0.3^{* \# \#}$ \\
\hline & 4 & $0.4 \pm 0.1$ & $0.3 \pm 0.1$ & $0.3 \pm 0.1$ & $2.3 \pm 0.8$ ****\#\#\# \\
\hline & 5 & $0.3 \pm 0.1$ & $0.5 \pm 0.2$ & $0.4 \pm 0.1$ & $1.9 \pm 0.7$ ***\#\# \\
\hline
\end{tabular}

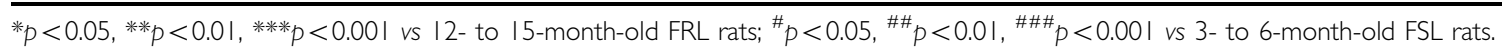

rapid glutamate spillover events compared with the sentinel-recording site. This release was only observed on the L-glutamate oxidase-coated sites and not on the selfreferencing recording sites, confirming that it was from spillover of glutamate and not noise or artifact. As shown in the trace, the spontaneous increases in glutamate spillover were robust compared with baseline, and occur intermittently throughout the duration of the experiment. The selfreferenced subtracted glutamate signal from this trace is shown in Figure 2e. Figures $2 \mathrm{~b}-\mathrm{e}$ show a series of representative traces of rapid glutamate transients in the 3- to 6-month-old FRL (2B) and FSL (2C), and the 12- to 15month-old FRL (2D) and FSL (2E) rats. Arrows beneath each trace indicate a glutamate transient. Each trace shows 4-5 glutamate transients during an approximate $100 \mathrm{~s}$ interval. As evidenced by the traces, the young FRL and FSL and the old FRL rats have similar glutamate-transient amplitudes. However, the old FSL rats show markedly increased concentrations of glutamate during these transient events. No change in animal behavior was observed during these transient events.

A large number of rapid glutamate transients were observed over the three testing days in the 3- to 6-month-old FRL (2241; $n=7)$ and FSL $(2041 ; n=6)$ and the 12- to 15-month-old FRL $(1235 ; n=4)$ and FSL $(1296 ; n=4)$ rats. Table 1 shows the average number of rapid glutamate transients per minute, transient interval, transient duration, and maximum amplitude of on each testing day for each rat group. A two-way ANOVA revealed that the day post-implantation had no effect on the number of rapid glutamate transients per minute $(\mathrm{F}=0.12 ; \mathrm{DFn}=2, \mathrm{DFd}=49 ; p=0.89)$, transient interval $(\mathrm{F}=0.07 ; \quad \mathrm{DFn}=2, \quad \mathrm{DFd}=49 ; p=0.93)$, transient duration $(\mathrm{F}=1.58 ; \mathrm{DFn}=2, \mathrm{DFd}=49 ; p=0.21)$, or transient amplitude $(\mathrm{F}=0.30 ; \mathrm{DFn}=2, \mathrm{DFd}=49 ; p=0.74)$. Among rat groups, no differences in rapid glutamate transients per minute $(\mathrm{F}=0.02 ; \mathrm{DFn}=2, \mathrm{DFd}=49 ; p=0.99)$ or transient interval $(\mathrm{F}=0.80 ; \mathrm{DFn}=3, \mathrm{DFd}=9 ; p=0.57)$ were observed. But transient duration $(\mathrm{F}=15.22 ; \quad \mathrm{DFn}=3, \quad \mathrm{DFd}=49$; $p<0.0001)$ and amplitude $(\mathrm{F}=23.90 ; \mathrm{DFn}=3, \mathrm{DFd}=49$; $p<0.0001)$ were significantly different among rat groups. As no interaction was observed for transient duration $(\mathrm{F}=1.30$; $\mathrm{DFn}=6, \mathrm{DFd}=49 ; p=0.27)$ or amplitude $(\mathrm{F}=0.55 ; \mathrm{DFn}=6$, $\mathrm{DFd}=49 ; p=0.77$ ), a Bonferonni post-hoc was used to determined that transient duration was increased in the 12- to 15-month-old FSL rats compared with age-matched controls $(p<0.001$, day $3 ; p<0.05$, day 4 post-implantation) and the 3- to 6-month-old FSL rats $(p<0.001$, day $3 ; p<0.01$, day 4 post-implantation). Also, the transient maximum amplitude in the 12- to 15-month-old FSL rats was significantly increased compared with age-matched controls $(p<0.05$ day $3, p<0.001$ day $4, p<0.01$ day 5 postimplantation) and the 3 - to 6 -month-old FSL rats $(p<0.01$ day $3, p<0.001$, day $4, p<0.01$, day 5 post-implantation).

As no differences in rapid glutamate transients were observed within rat groups over days, we averaged the results of days 3,4 , and 5 post-implantation into a single data point for each rat within a testing group as shown in Figure 3. Using a one-way ANOVA, no difference in the transient interval $(\mathrm{F}=1.49, \mathrm{df}=3, p=0.25)$ was observed in the 3 - to 6-month-old FRL $(61 \pm 9 \mathrm{~s})$ and FSL $(56 \pm 2 \mathrm{~s})$ and the 12 - to 15 -month-old FRL $(72 \pm 13 \mathrm{~s})$, and FSL $(82 \pm 15 \mathrm{~s})$ rats as shown in Figure $3 a$. The average transient duration was shown in Figure 3b. One-way ANOVA indicated a significant effect of transient duration $(\mathrm{F}=6.77 ; \mathrm{df}=3$; $p=0.003)$. A Tukey's post-hoc analysis revealed no differences in the transient duration of young FRL $(15 \pm 2 \mathrm{~s})$ and FSL $(15 \pm 3 s)$ rats, but the duration was significantly longer $(p<0.05)$ in the 12 - to 15 -month-FSL $(34 \pm 7 \mathrm{~s})$ compared with age-matched FRL ( $15 \pm 2 \mathrm{~s})$ rats and 3-to 6-month-old FSL rats $(p<0.01)$. Finally, the maximum amplitude of the rapid glutamate transients is shown in Figure 3c. One-way ANOVA indicated a significant difference $(\mathrm{F}=12.43, \mathrm{df}=3$, 

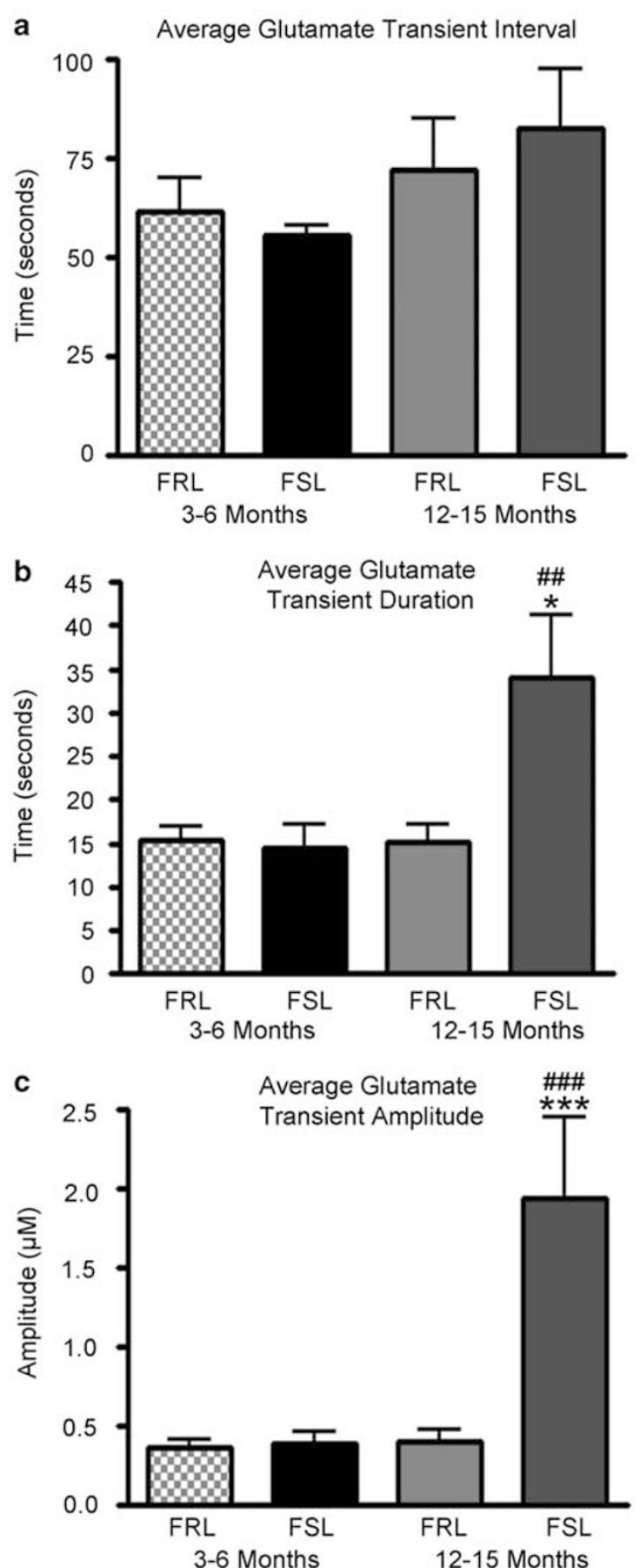

Figure 3 Analysis of rapid glutamate transients. The glutamate transient interval (a), duration (b), and maximum amplitude (c) were averaged across days post-implantation. On average, the inter-burst interval occurred twice per minute in all the four rat groups. The average burst duration was similar in the young FRL $(n=7)$ and FSL $(n=6)$ and the old FRL $(n=4)$ rats. But, a one-way ANOVA with a Tukey's post-hoc indicates that the 12- to I5-month-old FSL rats $(n=4)$ had significantly longer burst duration compared with age-matched FRL rats $(* p<0.05)$ and young FSL rats $\left({ }^{\# \#} p<0.0\right.$ I). Similar to burst duration, the average maximum amplitude of glutamate release was similar in the 3- to 6-month-old FRL and FSL and the 12- to 15-month-old FRL rats. Using the same calculations as (b), the 12- to I5-month-old FSL rats had significantly higher average concentrations of glutamate during spillover events compared with age-matched FRL rats (**** $p<0.00$ I) and 3- to 6-month-old FSL $\left({ }^{\# \#} p<0.00\right.$ I) rats. $p=0.0002)$ in the average maximum amplitude of glutamate transients. A Tukey's post-hoc analysis revealed no difference between 3- to 6-month-old FRL $(0.4 \pm 0.1 \mu \mathrm{M})$ and FSL $(0.4 \pm 0.1 \mu \mathrm{M})$ rats. However, the average maximum amplitude was significantly $(p<0.001)$ increased in the 12 - to 15-month-old FSL $(1.9 \pm 0.5 \mu \mathrm{M})$ rats compared with age-matched FRL $(0.4 \pm 0.1 \mu \mathrm{M})$ rats. Also, the average maximum amplitude of the rapid glutamate transients was significantly increased $(p<0.001)$ in the 12 - to 15 -monthold FSL rats compared with the 3- to 6-month-old FSL rats.

Similar glutamate transients were observed in the dentate gyrus of 3- to 6-month and 18-month-old Fischer-344 rats, a strain commonly used for aging studies and not a model of depression. Table 2 presents rapid glutamate transients in these rats compared with age-matched FSL rats on day 3 post-implantation. No differences in resting glutamate or rapid glutamate transients were observed between the 3- to 6-month and 18-month-old Fischer-344 rats. However, transient duration was increased in the 3- to 6-month $(t=4.8, \mathrm{df}=11 ; p<0.001)$ and 12 - to 15 -month $(t=3.7$, $\mathrm{df}=8 ; p<0.001)$-old FSL rats compared with age-matched Fischer-344 rats. Also, the amplitude of glutamate transients bursts was increased in the 12 - to 15 -month $(t=3.5, \mathrm{df}=8$; $p<0.01)$ compared with 18 -month-old Fischer-344 rats. Thus, our data support the effects that are likely unique to the FSL rats and not just to age-related changes in glutamate signaling.

\section{DISCUSSION}

An increasing body of evidence exists for the role of glutamate in depression. Thus, abnormal expression of genes involved in regulation of glutamate synthesis, uptake and metabolism, increased glutamate levels, and dysregulated function of glutamatergic receptors were suggested to be involved in pathogenesis and pathophysiology of depression. (Terracciano et al, 2010; McNally et al, 2008). To experimentally test the hypothesis of abnormal glutamate signaling in depression, we examined resting extracellular glutamate levels and the neurochemical activity of glutamate in the mPFC of awake FSL rat, a validated genetic model of depression. FSL rats were selectively bred from a Sprague-Dawley background, and have phenotypic traits similar to depressed individuals including decreased motivation, body weight (also observed in this study; data not shown), and psychomotor activity. These rats show severe immobility on the forced swim task that was reversible after chronic treatment with antidepressants (Overstreet et al, 2005).

The concept of glutamatergic dysregulation is an emerging field in depression research. Because of this, few studies of rodent models of depression exist for direct comparison. Takahashi et al (2011) demonstrated that a single dose of riluzole $(10 \mathrm{mg} / \mathrm{kg}$, p.o.) significantly decreased resting glutamate levels in the PFC of olfactory bulbectomized rats by in vivo microdialysis. Sartorius and colleagues (2007) used a model of learned helplessness combined with magnetic resonance spectroscopy and found an increased glutamate/ $\gamma$-aminobutyric acid (GABA) ratio that was returned to control levels with treatment of either desipramine $(10 \mathrm{mg} / \mathrm{kg}$, i.p.) or electroconvulsive therapy. 
Table 2 Rapid Glutamate Transients in the Dentate Gyrus of Fischer-344 rats

\begin{tabular}{|c|c|c|c|c|}
\hline Rat & Fischer $344(n=7)$ & FSL $(n=6)$ & Fischer $344(n=6)$ & FSL $(n=4)$ \\
\hline Age & 3- to 6-month-old & 3- to 6-month-old & |8-month-old & 12- to I5-month-old \\
\hline Resting glutamate $(\mu \mathrm{M})$ & $7.0 \pm 1.6$ & - & $12.2 \pm 4.2$ & - \\
\hline Glutamate transient interval $(\mathrm{s} \pm$ SEM) & $69.4 \pm 7.2$ & - & $100.0 \pm 20.0$ & - \\
\hline Glutamate transient duration ( $\mathrm{s} \pm \mathrm{SEM}$ ) & $2.9 \pm 0.5$ & $\uparrow \uparrow \uparrow$ & $6.3 \pm 1.8$ & $\uparrow \uparrow \uparrow$ \\
\hline Glutamate transient amplitude $(\mu \mathrm{M} \pm \mathrm{SEM})$ & $1.0 \pm 0.3$ & - & $0.4 \pm 0.2$ & $\uparrow \uparrow$ \\
\hline
\end{tabular}

Values for FSL day 3 post-implantation are located in Table I. Symbols indicate: -, no difference; $\uparrow \uparrow, p<0.0$ I; $\uparrow \uparrow \uparrow, p<0.00$ I.

Finally, Garcia et al (2009) have observed similar findings in a vesicular glutamate transporter 1 (vGluT1) knockout mouse model of depression. Using ${ }^{13} \mathrm{C}$ magnetic resonance spectroscopy, vGluT1 heterozygous mice also had an increased glutamate/GABA ratio. For a more thorough review of the pathophysiology of glutamatergic dysregulation in rodent models of depression the reader is referred to Mitchell and Baker (2010).

Although no differences in glutamatergic regulation were observed within each group, an intriguing finding from this study was that only the 12- to 15 -month-old FSL rat group showed differences in resting glutamate and rapid glutamate transients. This may indicate that FSL rats have a predisposition to depression as they age (Overstreet et al, 2005). In fact, Braw et al (2006) found that pre-pubertal (40-day old) FSL rats have lowered anxiety-like behaviors in the open-field and elevated plus mazes compared with age-matched FRL controls. By 3 months of age, FSL and FRL rats spend the same amount of time in the open areas of these behavioral tests. But in other behavioral studies, such as the social interaction and active avoidance tasks, 3-month-old FSL rats exhibit increased anxiogenic behavior compared with age-matched FRL rats (Overstreet et al, 2004; Overstreet et al, 1990). These studies raise the possibility of more drastic changes in the anxiety/depression profile of FSL rats as they continue to age (Braw et al, 2006); which may explain the why only 12 - to 15 -month-old FSL rats showed differences in resting and rapid glutamate transients. This time point (or earlier between 7 and 11 months) could mark a transition period from predisposition to depression, to depression, or even an elevated severity of anxiety/depression. In fact, Mitani et al (2006) showed that plasma levels of glutamate were positively correlated to the severity of depression in depressed individuals. To test this hypothesis, one would need to examine glutamate regulation in these rats at a more intermediate time span (between 7 and 11 months) and later in development (between 18 and 20 months).

This increase in extrasynaptic glutamate and rapid glutamate transients observed in the 12- to 15-month-old FSL rats could derive from any of several sources. Excessive synaptic stimulation may overwhelm the high-affinity glutamate uptake transporters located primarily on glia, thereby leading to the overflow of glutamate into the extracellular space (Pittenger et al, 2007). An alternate hypothesis has been proposed by Sanacora and colleagues (2005), based on imaging studies supporting a reduction of glia in the cortex of depressed patients. This could lead to fewer high-affinity glutamate transporters, thereby, reducing their ability to efficiently clear synaptic spillover of glutamate. Alternately, decreases in metabotropic autoreceptors could further increase spillover of glutamate. Several recent studies in the FSL rats support this line of reasoning. For example, a decrease in hippocampal volume was observed in FSL rats compared with control FRL rats (Chen et al, 2009). Matrisciano et al (2008) have also shown reduced expression and receptor signaling of the presynaptic, inhibitory $\mathrm{mGluR}_{2 / 3}$ autoreceptors in the hippocampus of FSL rats. Although these studies were performed in the FSL hippocampus, it has been shown that the rat PFC receives glutamatergic afferents from the hippocampus (Carr and Sesack, 1996). Loss of the $\mathrm{mGluR}_{2 / 3}$ receptors in the hippocampus could cause disinhibition of the glutamatergic efferents projecting to the PFC, leading to increased glutamate release, which we observe in resting glutamate as well as increased glutamate spillover in the 12- to 15-monthold FSL rats.

This is the first reported observations of rapid glutamate transients, and is a result of the high temporal, low spatial, and minimal damage of the MEAs. Our laboratory has also recorded these rapid glutamate transients in the hippocampus of awake Fischer-344 rats, a strain typically used in aging studies. We also have data to support that these signals are greatly affected by TTX. Furthermore, we previously reported that local application of either TTX or $\omega$-conotoxin (MVIIC; calcium channel blocker) reduces resting glutamate levels by $40-50 \%$ in the PFC of freely behaving rats, as well as abolishes the glutamatergic response from a tail-pinch stressor (Hascup et al, 2010). As recent studies support that gliotransmission was not calcium dependent (Agulhon et al, 2010), this data suggests a possible neuronal origin for the rapid glutamate transients reported in this manuscript.

The main finding from this study regarding increased resting glutamate levels in the 12- to 15-month-old FSL rats was similar to previous reports of glutamatergic alterations in depressed patients as previously discussed. An important consideration of all animal models is the translation to the human condition, which is dependent upon the animal model's validity. In fact, an animal model that perfectly matches the human condition is highly unlikely, and therefore, emphasis on animal models of disease should focus on specific components (Malkesman and Weller, 2009). For example, studies support that the FSL rat is not relevant to the norepinephrine model of depression, and the data consistent with the serotonin and dopamine models are mixed (Overstreet et al, 2005). Our data supports that only 12- to 15-month-old FSL rats might be relevant to 
the glutamate model of depression. Thus, the results of the present studies should be investigated in addition to animal models of depression, but the present studies point to the phasic changes in the glutamate system in this model that may be highly relevant to the treatment of the human condition and to the effects of chronic, untreated, or undiagnosed depression.

\section{ACKNOWLEDGEMENTS}

This work was supported by the NSF EEC-0310723; NIH/NIDA DA017186; CEBRA, Phase II (GA Gerhardt), and grants from the Swedish Foundation for Strategic Research (SSF, R98:022) and the European 6FP program (Biodiagnostics, NMP4-CT-2005-017002) (J Kehr), and the Swedish Medical Research Council grant 10414 (AA Mathé).

\section{DISCLOSURE}

Greg A Gerhardt is the sole proprietor of Quanteon, LLC. Jan Kehr is the sole proprietor of Pronexus Analytical AB. The other authors declare no conflict of interest.

\section{REFERENCES}

Agulhon C, Fiacco TA, McCarthy KD (2010). Hippocampal shortand long-term plasticity are not modulated by astrocyte $\mathrm{Ca}^{2+}$ signaling. Science 327: 1250-1254.

Altamura CA, Mauri MC, Ferrara A, Moro AR, D'Andrea G, Zamberlan F (1993). Plasma and platelet excitatory amino acids in psychiatric disorders. Am J Psychiatry 150: 1731-1733.

Berman RM, Cappiello A, Anand A, Oren DA, Heninger GR, Charney DS et al (2000). Antidepressant effects of ketamine in depressed patients. Biol Psychiatry 47: 351-354.

Braw Y, Malkesman O, Dagan M, Bercovich A, Lavi-Avnon Y, Schroeder M et al (2006). Anxiety-like behaviors in pre-pubertal rats of the flinders sensitive line (FSL) and Wistar-Kyoto (WKY) animal models of depression. Behav Brain Res 167: 261-269.

Brennan BP, Hudson JI, Jensen JE, McCarthy J, Roberts JL, Prescot AP et al (2010). Rapid enhancement of glutamatergic neurotransmission in bipolar depression following treatment with riluzole. Neuropsychopharmacology 35: 834-846.

Burmeister JJ, Coates TD, Gerhardt GA (2004). Multisite microelectrode arrays for measurements of multiple neurochemicals. Conf Proc IEEE Eng Med Biol Soc 7: 5348-5351.

Burmeister JJ, Gerhardt GA (2001). Self-referencing ceramic-based multisite microelectrodes for the detection and elimination of interferences from the measurement of L-glutamate and other analytes. Anal Chem 73: 1037-1042.

Burmeister JJ, Moxon K, Gerhardt GA (2000). Ceramic-based multisite microelectrodes for electrochemical recordings. Anal Chem 72: 187-192.

Burmeister JJ, Pomerleau F, Palmer M, Day BK, Huettl P, Gerhardt GA (2002). Improved ceramic-based multisite microelectrode for rapid measurements of L-glutamate in the CNS. J Neurosci Methods 119: 163-171.

Canbeyli R (2010). Sensorimotor modulation of mood and depression: an integrative review. Behav Brain Res 207: 249-264.

Carr DB, Sesack SR (1996). Hippocampal afferents to the rat prefrontal cortex: synaptic targets and relation to dopamine terminals. J Comp Neurol 369: 1-15.

Chen F, Madsen TM, Wegener G, Nyengaard JR (2009). Imipramine treatment increases the number of hippocampal synapses and neurons in a genetic animal model of depression. Hippocampus 2010: 1376-1384.
Danbolt NC (2001). Glutamate uptake. Prog Neurobiol 65: 1-105.

Garcia-Garcia AL, Elizalde N, Matrov D, Harro J, Wojcik SM, Venzala E et al (2009). Increased vulnerability to depressive-like behavior of mice with decreased expression of VGLUT1. Biol Psychiatry 66: 275-282.

Hascup ER, af BS, Hascup KN, Pomerleau F, Huettl P, Stromberg I et al (2009). Histological studies of the effects of chronic implantation of ceramic-based microelectrode arrays and microdialysis probes in rat prefrontal cortex. Brain Res 1291: 12-20.

Hascup ER, Hascup KN, Stephens M, Pomerleau F, Huettl P, Gratton A et al (2010). Rapid microelectrode measurements and the origin and regulation of extracellular glutamate in rat prefrontal cortex. J Neurochem 115: 1608-1620.

Hascup KN, Hascup ER, Pomerleau F, Huettl P, Gerhardt GA (2008). Second-by-second measures of L-glutamate in the prefrontal cortex and striatum of freely moving mice. J Pharmacol Exp Ther 324: 725-731.

Hascup KN, Rutherford EC, Quintero JE, Day BK, Nickell JR, Pomerleau F, Huettl P, Burmeister JJ, Gerhardt GA (2006). Second-by-second measures of L-glutamate and other neurotransmitters using enzyme-based microelectrode arrays. In: Michael AC and Borland LM (eds). Electrochemical Methods for Neuroscience. CRC Press: Boca Raton FL. pp 407-450.

Hashimoto K, Sawa A, Iyo M (2007). Increased levels of glutamate in brains from patients with mood disorders. Biol Psychiatry 62: 1310-1316.

Insel TR, Wang PS (2009). The $\operatorname{STAR}^{\star} \mathrm{D}$ trial: revealing the need for better treatments. Psychiatr Serv 60: 1466-1467.

Kim JS, Schmid-Burgk W, Claus D, Kornhuber HH (1982). Increased serum glutamate in depressed patients. Arch Psychiatr Nervenkr 232: 299-304.

Kugaya A, Sanacora G (2005). Beyond monoamines: glutamatergic function in mood disorders. CNS Spectr 10: 808-819.

Kulkarni SK, Dhir A (2009). Current investigational drugs for major depression. Expert Opin Investig Drugs 18: 767-788.

Li N, Lee B, Liu RJ, Banasr M, Dwyer JM, Iwata M et al (2010). mTOR-dependent synapse formation underlies the rapid antidepressant effects of NMDA antagonists. Science 329: 959-964.

Maeng S, Zarate Jr CA (2007). The role of glutamate in mood disorders: results from the ketamine in major depression study and the presumed cellular mechanism underlying its antidepressant effects. Curr Psychiatry Rep 9: 467-474.

Malkesman O, Weller A (2009). Two different putative genetic animal models of childhood depression-a review. Prog Neurobiol 88: 153-169.

Matrisciano F, Caruso A, Orlando R, Marchiafava M, Bruno V, Battaglia $G$ et al (2008). Defective group-II metaboropic glutamate receptors in the hippocampus of spontaneously depressed rats. Neuropharmacology 55: 525-531.

Mauri MC, Ferrara A, Boscati L, Bravin S, Zamberlan F, Alecci M et al (1998). Plasma and platelet amino acid concentrations in patients affected by major depression and under fluvoxamine treatment. Neuropsychobiology 37: 124-129.

McCauley E, Myers K, Mitchell J, Calderon R, Schloredt K, Treder $R$ (1993). Depression in young people: initial presentation and clinical course. J Am Acad Child Adolesc Psychiatry 32: 714-722.

McNally L, Bhagwagar Z, Hannestad J (2008). Inflammation, glutamate, and glia in depression: a literature review. CNS Spectr 13: $501-510$.

Mitani H, Shirayama Y, Yamada T, Maeda K, Ashby Jr CR, Kawahara R (2006). Correlation between plasma levels of glutamate, alanine and serine with severity of depression. Prog Neuropsychopharmacol Biol Psychiatry 30: 1155-1158.

Mitchell ND, Baker GB (2010). An update on the role of glutamate in the pathophysiology of depression. Acta Psychiatr Scand 122: 192-210. 
Nickell J, Pomerleau F, Allen J, Gerhardt GA (2005). Age-related changes in the dynamics of potassium-evoked L-glutamate release in the striatum of Fischer 344 rats. J Neural Transm 112: 87-96.

Nierenberg AA (2001). Do some antidepressants work faster than others? J Clin Psychiatry 62(Suppl 15): 22-25.

Overstreet DH (1993). The Flinders sensitive line rats: a genetic animal model of depression. Neurosci Biobehav Rev 17: 51-68.

Overstreet DH, Friedman E, Mathe AA, Yadid G (2005). The Flinders sensitive line rat: a selectively bred putative animal model of depression. Neurosci Biobehav Rev 29: 739-759.

Overstreet DH, Janowsky DS, Pucilowski O, Rezvani AH (1994). Swim test immobility co-segregates with serotonergic but not cholinergic sensitivity in cross-breeds of Flinders line rats. Psychiatr Genet 4: 101-107.

Overstreet DH, Keeney A, Hogg S (2004). Antidepressant effects of citalopram and CRF receptor antagonist CP-154,526 in a rat model of depression. Eur J Pharmacol 492: 195-201.

Overstreet DH, Rezvani AH, Janowsky DS (1990). Impaired active avoidance responding in rats selectively bred for increased cholinergic function. Physiol Behav 47: 787-788.

Overstreet DH, Russell RW (1982). Selective breeding for diisopropyl fluorophosphate-sensitivity: behavioural effects of cholinergic agonists and antagonists. Psychopharmacology (Berl) 78: $150-155$

Paul IA, Skolnick P (2003). Glutamate and depression: clinical and preclinical studies. Ann N Y Acad Sci 1003: 250-272.

Paxinos G, Watson C. (1998). The Rat Brain: Stereotaxic Coordinates. Academic Press: New York.

Pittenger C, Sanacora G, Krystal JH (2007). The NMDA receptor as a therapeutic target in major depressive disorder. CNS Neurol Disord Drug Targets 6: 101-115.

Pollak DD, Rey CE, Monje FJ (2010). Rodent models in depression research: classical strategies and new directions. Ann Med 42: 252-264.
Rakofsky JJ, Holtzheimer PE, Nemeroff CB (2009). Emerging targets for antidepressant therapies. Curr Opin Chem Biol 13: 291-302.

Rutherford EC, Pomerleau F, Huettl P, Stromberg I, Gerhardt GA (2007). Chronic second-by-second measures of L-glutamate in the central nervous system of freely moving rats. J Neurochem 102: $712-722$

Sanacora G, Kendell SF, Levin Y, Simen AA, Fenton LR, Coric V et al (2007). Preliminary evidence of riluzole efficacy in antidepressant-treated patients with residual depressive symptoms. Biol Psychiatry 61: 822-825.

Sartorius A, Mahlstedt MM, Vollmayr B, Henn FA, Ende G (2007). Elevated spectroscopic glutamate/gamma-amino butyric acid in rats bred for learned helplessness. Neuroreport 18: 1469-1473.

Skolnick P, Popik P, Trullas R (2009). Glutamate-based antidepressants: 20 years on. Trends Pharmacol Sci 30: 563-569.

Stone M, Laughren T, Jones ML, Levenson M, Holland PC, Hughes A et al (2009). Risk of suicidality in clinical trials of antidepressants in adults: analysis of proprietary data submitted to US food and drug administration. BMJ 339: b2880.

Takahashi K, Murasawa H, Yamaguchi K, Yamada M, Nakatani A, Yoshida $\mathrm{M}$ et al (2011). Riluzole rapidly attenuates hyperemotional responses in olfactory bulbectomized rats, an animal model of depression. Behav Brain Res 216: 46-52.

Terracciano A, Tanaka T, Sutin AR, Sanna S, Deiana B, Lai S et al (2010). Genome-wide association scan of trait depression. Biol psychiatry 68: 811-817.

Witkin JM, Marek GJ, Johnson BG, Schoepp DD (2007). Metabotropic glutamate receptors in the control of mood disorders. CNS Neurol Disord Drug Targets 6: 87-100.

Yadid G, Nakash R, Deri I, Tamar G, Kinor N, Gispan I et al (2000). Elucidation of the neurobiology of depression: insights from a novel genetic animal model. Prog Neurobiol 62: 353-378. 